\title{
LA PRONUNCIACIÓN DE LOS PROFESORES DE ESPAÑOL COMO LENGUA EXTRANJERA Y SEGUNDA LENGUA: LA REALIDAD DEL AULA.
}

\author{
Tania Silverio Pérez \\ Wang Ye ${ }^{* *}$ \\ Caridad Abreu López*** \\ http://orcid.org/0000-0002-2379-6713
}

RECIBIDO: Agosto 2019 / ACEPTADO: Marzo 2020 / PUBLICADO: Mayo 2020

Como citar: Silverio Pérez, Tania; Ye, Wang; Abreu López, Caridad (2020). La pronunciación de los profesores de español como lengua extranjera y segunda lengua: la realidad del aula. Telos: revista de Estudios Interdisciplinarios en Ciencias Sociales, 22 (2), Venezuela. (Pp.357-375).

DOI: www.doi.org/10.36390/telos222.08

\section{RESUMEN}

En el contexto docente, el profesor es el modelo de lengua más importante del estudiante de lenguas extranjeras, sobre todo en los niveles iniciales. Las exigencias en relación con la pronunciación, si se considera el primer contacto con la lengua extranjera y la adaptación de su oído fonemático, constituyen un gran reto para el estudiante. El objetivo de este trabajo es describir la pronunciación del profesor en las clases de español como lengua extranjera y segunda lengua, de los niveles A1 y A2, en la Facultad de Español para No Hispanohablantes de la Universidad de La Habana. Sirvieron de referencia algunas investigaciones como las de Lahera (2011), Silverio (2014), Mingjin (2016) y Wang (2018). Se trabajó con muestras de lengua oral de cinco docentes, obtenidas durante clases de nivel principiante, pues la esfera académica es la que interesa a los fines de esta investigación. Se realizó la descripción articulatoria a partir de la percepción auditiva, y el software Praat se empleó para la transcripción de la grabación y el análisis de aquellos segmentos de la cadena hablada en los que la percepción sugería que había variaciones. El análisis demostró que la pronunciación se caracteriza por la estabilidad en la realización de los sonidos; los informantes tienden a fragmentar el enunciado a favor de la comprensión de los estudiantes, lo que limita la pronunciación correcta de las palabras fónicas, pues desaprovechan posibilidades de sinalefa y enlaces consonante-vocal, propios de las palabras fónicas. Los principales procesos de variación están relacionados con los fonemas /s/ $\mathrm{y} / \mathrm{d} /$.

Palabras clave: enseñanza, español, lengua extranjera, pronunciación, profesor

\footnotetext{
Departamento de Estudios Lingüísticos y Culturales, Facultad de Español para No Hispanohablantes de la Universidad de La Habana. Correo electrónico: tania.sp@fenhi.uh.cu

Máster en Estudios Lingüísticos del Español como Lengua Extranjera, Facultad de Español para No Hispanohablantes de la Universidad de La Habana. Correo electrónico: wywy 1984@163.com

*** Departamento de Relaciones Internacionales. Facultad de Ciencias Sociales y Humanas. Universidad Oscar Ribas. Luanda, Angola. Correo electrónico: carypa2013@gmail.com
} 
La pronunciación de los profesores de español como lengua extranjera y segunda lengua: la realidad del aula.

\title{
The pronunciation of the teachers of spanish foreign language and second language: the reality of the classroom
}

\begin{abstract}
In the teaching context, especially at the initial levels, the most important language model of the foreign language student is the teacher. The requirements in relation to pronunciation, if the first teacher's contact with the foreign language and the adaptation of phonemic ear are considered, constitute a great challenge for the student. The objective of this work is to describe the pronunciation of the teacher in the classes of Spanish as a foreign language and second language, of levels $A 1$ and A2, in the Faculty of Spanish for Non-Spanish speakers of the University of Havana. Reference was made to some research such as Lahera (2011), Silverio (2014), Mingjin (2016) and Wang (2018). It was worked with samples of oral language from five teachers, obtained during beginner level classes, because the academic sphere is the one that interests the purpose of this research. The articulatory description was made from the auditory perception, and the Praat software was used for the transcription of the recording and the analysis of those segments of the spoken chain in which the perception suggested that there were variations. The analysis showed that pronunciation is characterized by stability in the realization of sounds; informants tend to fragment the statement in favor of student understanding, which limits the correct pronunciation of phonic words, because they miss out on possibilities of synalefa and consonant-vocal links, typical of phonic words. The main variation processes are related to the phonemes $/ \mathrm{s} /$ and $/ \mathrm{d} /$.
\end{abstract}

Keywords: teaching, Spanish, foreign language, pronunciation, teacher

\section{Introducción}

Para quienes consideran que la adquisición fónica es anterior a cualquier adquisición léxico-gramatical (Cantero, 2003), la adquisición fónica constituye el primer gran reto del estudiante cuando aprende una lengua extranjera (LE), pues es muy difícil automatizar un conjunto de gestos articulatorios nuevos o que han resultado de la modificación de los ya conocidos.

Desde el punto de vista didáctico, la pronunciación es:

una habilidad que ha de enseñarse y desarrollarse en el proceso de enseñanzaaprendizaje del ELE 1 para que el estudiante, como parte de su competencia comunicativa, alcance una competencia fónica, es decir, la capacidad para utilizar la dimensión fónica de la lengua con eficacia en las diferentes situaciones comunicativas (Silverio, 2014: 14).

Existen varios trabajos sobre la pronunciación del español como lengua extranjera (ELE) y segunda lengua (L2) en el contexto cubano. Pueden ser mencionados, por ejemplo, en la Universidad de La Habana las investigaciones de Silverio (2007), Lahera (2011), y Mingjin (2016), quienes se centran en la descripción acústico-articulatoria de la realización de

\footnotetext{
${ }^{1}$ La autora se refiere al español como lengua extranjera (ELE).
} 
segmentos fónicos del español por sino hablantes. Estas investigaciones lingüísticas refieren aspectos de la interlengua fónica en el proceso de aprendizaje del español.

En el caso de Hernández (2011), la autora expone un sistema de ejercicios basado en la utilización del karaoke como herramienta motivadora para el mejoramiento de la pronunciación de los estudiantes chinos de segundo año de la Licenciatura en Español para No Hispanohablantes; Wei, Dell y Sun (2013), sugieren un conjunto de ejercicios, también para la corrección fonética, pero para estudiantes chinos de tercer año de Pedagogía en Humanidades; y Wei (2016), presenta un conjunto de actividades tipo para perfeccionar la pronunciación a fin de contribuir al desarrollo de la expresión oral en la asignatura Lengua y Comunicación I, también en la Licenciatura en Español para No Hispanohablantes.

En las investigaciones referidas se mencionan las dificultades de pronunciación de los estudiantes, las carencias de los programas y de las clases en relación con esta habilidad, de forma general, pero el análisis desde la función del profesor en el proceso es limitado.

Silverio (2014), cuando refiere los resultados de la observación de clases, de nivel principiante del Curso de Corta Duración del Departamento de Español de la Facultad de Lenguas Extranjeras (FLEX), para el diagnóstico de su investigación, plantea que:

El profesor no siempre es un modelo lingüístico óptimo, aunque sea un exponente suficiente de su lengua. De los cuatro profesores, dos fragmentaban su discurso para hacerse entender, por lo que el ritmo, la entonación y, en ocasiones, el acento, se presentaban en un modelo falso. (p. 66)

Esta autora propone una estrategia linguodidáctica para perfeccionar la enseñanza de la pronunciación en los Cursos de Corta Duración que se impartían en la FLEX, y ahora en la Facultad de Español para No Hispanohablantes (FENHI). Parte de la preparación del profesor, pues es quien guía científicamente el proceso de aprendizaje del estudiante mediante la creación de ambientes que propicien la formación desde una influencia educativa intencionada, científica y que respeta las regularidades del desarrollo del ser humano, de su personalidad. Los profesores son un modelo, en tanto, son la figura que posee el conocimiento, pero también la forma ideal de ver, razonar y reaccionar ante la vida.

Uno de los anexos del trabajo de Silverio (2014), es un folleto con la descripción de la pronunciación de la variedad cubana del español, que concluye con el acápite «Decisiones relacionadas con la pronunciación para nivel principiante», en el que sugiere cómo debe ser el modelo lingüístico que se le presente al estudiante en el nivel principiante.

La FENHI tiene un proyecto científico cuyo objetivo es perfeccionar la didáctica de la pronunciación en las clases de ELE y L2, lo que ha repercutido favorablemente en el proceso de enseñanza-aprendizaje, pero aún no se ha comprobado si los profesores que trabajan los niveles iniciales han asumido su función como modelo lingüístico, según la propuesta de Silverio (2014), ni se ha validado dicha propuesta.

En la FENHI existen tres modalidades de estudio estables que trabajan los niveles iniciales de lengua: la Licenciatura en Lengua Española para No Hispanohablantes, la Preparatoria (curso de acceso a la universidad para no hispanohablantes), y los Cursos de Corta Duración, pertenecientes a la modalidad 2, que se enmarca en el turismo especializado en la rama educacional (aprendizaje de una lengua extranjera en el país donde se habla), y tienen 
La pronunciación de los profesores de español como lengua extranjera y segunda lengua: la realidad del aula.

como objetivo que el estudiante se comunique oralmente con la comunidad hispanohablante que lo rodea para satisfacer sus necesidades (Puig, 2004).

Cuando se habla de niveles iniciales de la lengua se hace referencia a los niveles A1 y A2 definidos por el Marco Común Europeo de Referencia para las Lenguas (MCER), con las adecuaciones pertinentes para cada modalidad de estudio en el contexto cubano, específicamente, en la Facultad.

En cuanto a especificaciones para los niveles A1 y A2, el MCER (2002: 111) expone:

A1: Su pronunciación, de un repertorio muy limitado de palabras y frases aprendidas, la pueden comprender con cierto esfuerzo los hablantes nativos acostumbrados a tratar con hablantes del mismo grupo lingüístico al que pertenece el usuario 0 alumno.

A2: Su pronunciación es generalmente, bastante clara y comprensible, aunque resulte evidente su acento extranjero y los interlocutores tengan que pedir repeticiones de vez en cuando.

Las exigencias para estos niveles, en relación con la pronunciación, parecieran ser pocas, pero si se considera que es el primer contacto del estudiante con la lengua extranjera y el momento de iniciar la adaptación de su oído fonemático a ella, constituyen un gran reto para los profesores y los estudiantes implicados.

La enseñanza del ELE y L2 en la Universidad de La Habana tiene en cuenta lo referido en el Marco Común Europeo de Referencia para las Lenguas y lo planteado por el Instituto Cervantes. Ambos documentos rigen la enseñanza del ELE internacionalmente. La escuela cubana toma lo positivo de ellos y lo adecua a su contexto.

Cuba pertenece al área geolectal del Caribe hispánico, por lo que comparte muchos rasgos con esa región, pero se identifica dentro de esta comunidad lingüística por algunos elementos, fundamentalmente fonéticos y léxicos. También entre las distintas zonas del país se pueden notar diferencias en la forma de hablar, pero esto no afecta el reconocimiento de la variedad cubana del español (VCE), que es la que se enseña.

El español usual en Cuba se caracteriza por su carácter unitario, evidente en la presencia de peculiaridades de la lengua comunes a todas las provincias. La investigadora Montero Bernal (2007), considera que, de acuerdo con esto, se puede corroborar la inexistencia de dialectos en la VCE.

El claustro de la FENHI está formado por profesores de diferentes partes del país. En este contexto es pertinente preguntarse: ¿qué modelo lingüístico tiene el estudiante en la figura del profesor en la Facultad de Español para No Hispanohablantes?

Tomando en cuenta esta pregunta, el objetivo de este trabajo es describir la pronunciación del profesor en las clases español como lengua extranjera y segunda lengua de los niveles A1 y A2, en la Facultad de Español para No Hispanohablantes.

A continuación se contextualiza la pronunciación de la VCE dentro del Caribe hispánico y se ofrecen algunas características que la tipifican. Esto constituye el punto de partida para, en apartados posteriores, exponer cómo debe ser la pronunciación del profesor en la clase de ELE y L2, y justificar la selección de los fonemas para el estudio. 
El acápite dedicado a la metodología da cuenta del proceso de definición del corpus y el procedimiento para su análisis. Luego se ofrecen características generales de la pronunciación de las informantes y el comportamiento de los fonemas objeto de estudio.

\section{La pronunciación de la variedad cubana del español (VCE)}

El español de América es un complejo mosaico de variedades que responden a factores históricos y sociales de cada país. El español del Caribe tiene mucho de los dialectos andaluz y canario, debido a los frecuentes arribos de barcos de Sevilla y las Islas Canarias. De esta forma, las tierras altas, que suelen tener pronunciaciones más conservadoras, se oponen a las bajas como las costeñas del Caribe, que suelen expresar una variedad radical de pronunciación, descrita por los procesos de elisión de consonantes.

«Las Antillas y las Bahamas constituyen una región insular desde el punto de vista geográfico, cuya cultura es perceptiblemente suramericana. Algunos autores han denominado esta área cultural "caribeña"». Valdés (2015: 89). El español caribeño es un conjunto de variedades diatópicas de la lengua española, predominante en las Antillas Mayores y la Cuenca del Caribe.

Cuba pertenece al área del Caribe hispánico, por lo que comparte muchos rasgos con esa región, pero se individualiza dentro de esta comunidad lingüística por algunos elementos, fundamentalmente fonéticos y léxicos. También entre las distintas zonas del país se pueden notar diferencias en la forma de hablar, pero esto no afecta el reconocimiento de la variedad cubana del español.

El español usual en Cuba se caracteriza por su carácter unitario, evidente en la presencia de peculiaridades de la lengua comunes a todas las provincias. La investigadora Montero Bernal (2007), explica que, de acuerdo con esto, se puede corroborar la inexistencia de dialectos en la VCE.

La revisión de bibliografía especializada en el tema ${ }^{2}$-Isbasescu (1968), Tristá y Valdés (1978), Costa y Carreras (1980), Choy (1988), Costa (1993), Dohotaru (1998/1999), García (1980), Silverio (2007, 2014); estudios referidos a la pronunciación panhispánica como Quilis (1993), D'Introno, del Teso y Weston (1995), Gil (2007) y Real Academia Española y Asociación de Academias de la Lengua Española (2013) - permite concluir que, de forma general, la pronunciación de la variedad cubana del español se identifica por fenómenos como el seseo, el yeísmo, la pérdida o aspiración de /-s/ y diversos procesos de debilitamiento de / $/ \mathrm{f}$ final absoluta de los infinitivos y otras palabras, realizaciones alternas con $/ /$, pérdida de /d/ intervocálica y velarización de la $/ \mathrm{n} /$ en final de sílaba. Estos cambios o variaciones están relacionados, en cierta medida, con una tendencia generalizada al relajamiento o disminución de la tensión articulatoria en todas las hablas hispánicas (Silverio, 2014), y condicionan cierto alargamiento vocálico.

\footnotetext{
${ }^{2}$ Se hace referencia a bibliografía que aborda aspectos segmentales de la pronunciación (fonemas y fonos), pues es la que interesa a los fines de este trabajo. Es evidente que los estudios sobre la VCE son insuficientes, en lo segmental y en lo suprasegmental -aunque este segundo aspecto se ha visto favorecido a partir de la obra de la entonóloga cubana Raquel García Riverón, sobre todo a partir de la incorporación de investigadores la Facultad de Humanidades de la Universidad Central Martha Abreu de Las Villas, en 2000.
} 
La pronunciación de los profesores de español como lengua extranjera y segunda lengua: la realidad del aula.

Se manifiestan diferencias regionales, sexuales y generacionales; sin embargo, existen rasgos generales que matizan el español que hablan los cubanos y le aportan un toque de singularidad. ${ }^{3}$

\section{La pronunciación del profesor de español como lengua extranjera (ELE) y segunda lengua (L2), niveles A1 y A2.}

El profesor debe ser un representante de la norma culta de la lengua española que enseña, independientemente del método y el enfoque empleados en la enseñanza de una lengua extranjera, en un contexto formal y presencial de aprendizaje, el profesor es el primer y más importante modelo de lengua para el estudiante

Silverio (2014), propone una estrategia linguodidáctica para perfeccionar la enseñanza de la pronunciación en los Cursos de Corta Duración que se impartían en la FLEX, y ahora, en la FENHI.

La autora parte de la preparación del profesor, pues es quien guía científicamente el proceso de aprendizaje del estudiante mediante la creación de ambientes que propicien la formación desde una influencia educativa intencionada, científica y que respeta la regularidad del desarrollo del ser humano, de su personalidad.

En esta concepción el profesor no es un facilitador sino un orientador, director, guía del proceso de aprendizaje.

Declara la autora:

Muchas veces los hablantes nativos de una lengua rechazan a quienes la aprenden como LE y, al emplearla, pretenden imitarlos, repetir sus expresiones "censuradas socialmente" y fenómenos que no se identifican con una imagen de prestigio de esa lengua. Pueden considerarlo irrespetuoso e inapropiado, aun cuando se corresponda con el contexto real para su uso. Para que el estudiante pueda desenvolverse eficientemente (de forma inteligible) en diferentes situaciones comunicativas en el medio lingüístico de inmersión -zona dialectal I, Cuba - es necesario enseñarle la norma culta (o estándar). La pronunciación debe despojarse, en esencia, de algunos fenómenos propios del registro familiar y popular (Silverio, 2014: 41-42).

Esta consideración lleva a la autora a plantear que en el modelo lingüístico que se le presenta al estudiante se deben evitar las elisiones de /-s/ final y no llevar las aspiraciones a una presencia absoluta, pues el oído fonemático del no hispanohablante no está preparado para percibirlas; se debe evitar el trueque de/r/por ///, de /// por/r/ y la pérdida de estos fonemas, ya que se puede provocar malentendidos en la comunicación; se deben evitarlas asimilaciones en contacto regresivo y mantenerse la /d/ intervocálica, lo que favorece la realización aproximante. En español, las vocales no constituyen una gran barrera, debido al número reducido de sus componentes y su estabilidad en el sistema. Siempre que el hablante mantenga el timbre que identifica la vocal, los procesos de variación posibles no repercuten en el reconocimiento del fonema.

\footnotetext{
${ }^{3}$ En este apartado no se hará referencia a las peculiaridades de la pronunciación de cada zona dialectal. Se retomará este tema, según las necesidades que exija el análisis de la muestra.
} 
El profesor de ELE se encuentra con la paradoja de tener que mostrar a sus alumnos una representación idealizada de la lengua meta a partir de su realización individual, sujeta siempre a rasgos concretos de una variante geográfica.

Gil (2007), considera que no es conveniente referirse a la pronunciación del español sin más precisiones y que la elección de un modelo u otro debe sustentarse en factores más objetivos que la supuesta mayor 0 menor pureza, prestigio 0 tradición de las diversas variedades geográficas.

Esta autora refiere la definición que Halliday et al. (1964: 296, citado por Gil, 2007), Dan de norma estándar: la variedad utilizada por un conjunto razonablemente amplio de la población total de los hablantes de la lengua y de personas cuyo nivel cultural los convierte en referencia deseable; mutuamente inteligible con otras variedades. También adopta el concepto aceptabilidad, introducido por Dalbor (1969, citado por Gil, 2007): «cualidad del dialecto; inteligible y relativamente poco notorio o menos marcado». Esta propiedad flexibiliza la noción de norma estándar o neutra.

Gil (2007), le atribuye mayor importancia a la realidad del entorno en que se enseña, Las necesidades de los estudiantes y la finalidad para la que se enseña a la hora de diseñar un curso, y recomienda usar materiales que reflejen, según las posibilidades y el nivel, la diversidad de acentos de la comunidad hispana. El profesor debe atender la relación pronunciación - registro; es decir, las correlaciones entre los tipos de discurso y los códigos sociales, para mostrarle al estudiante el español real.

El estudiante debe recibir la norma que use el profesor, la de los medios de comunicación a los que tenga acceso y la que hable la comunidad lingüística en la que esté inmerso, si estudian en un país hispanohablante. Es decir, la situación comunicativa en la que se desarrolla el aprendizaje determina la norma que, aunque no quede planteada, responde a una norma estándar. La introducción de los registros se justifica en los diferentes grados de formalidad en la pronunciación que requieran las situaciones comunicativas que se presenten en el aula.

El aula es un espacio intercultural, reflejo del mundo real que encontrarán los alumnos cuando salgan de clase e interactúen con hispanohablantes, de ahí que es primordial que el profesor sea consciente de las variaciones de cultura comunicativa y del origen de los malentendidos culturales.

\section{Aspectos que se deben revisar en la pronunciación del profesor}

En la Facultad de Español para No Hispanohablantes de la Universidad de La Habana hay profesores de varias provincias cubanas; por lo tanto, cuando desarrollan sus clases, aparecen características propias de sus lugares de origen, sobre todo en la pronunciación (en lo segmental y en lo suprasegmental) y el vocabulario (especialmente en el fraseologismo), a pesar del «reajuste» de la forma de hablar de cada uno tras un tiempo de inmersión (variable en ese claustro), y del carácter unitario que concede la norma culta de la lengua, pues un profesor siempre es, en teoria, un representante de la norma culta de su lengua materna.

Las reflexiones expuestas en apartados anteriores sugieren que la descripción de la pronunciación de los profesores que trabajan el ELE y L2 debe hacer hincapié en los siguientes fonemas: 
La pronunciación de los profesores de español como lengua extranjera y segunda lengua: la realidad del aula.

Tabla 1. Fonemas objeto de estudio y sus características articulatorias

\begin{tabular}{|c|c|c|c|c|}
\hline Fonemas & $\begin{array}{c}\text { Modo de } \\
\text { articulación }\end{array}$ & $\begin{array}{c}\text { Zona de } \\
\text { articulación }\end{array}$ & $\begin{array}{c}\text { Acción de } \\
\text { las cuerdas } \\
\text { vocales }\end{array}$ & $\begin{array}{c}\text { Acción del velo } \\
\text { del paladar }\end{array}$ \\
\hline$/ \mathrm{d} /$ & oclusivo & apicodental & sonoro & oral \\
\hline$/ \mathrm{s} /$ & fricativo & predorsoalveolar & sordo & oral \\
\hline$/ / /$ & Lateral & apicoalveolar & sonoro & oral \\
\hline$/ \mathrm{r} /$ & vibrante $(\mathrm{s})$ & apicoalveolar & sonoro & oral \\
\hline$/ \mathrm{r} /$ & vibrante $(\mathrm{m})$ & apicoalveolar & sonoro & oral \\
\hline
\end{tabular}

Fuente: Elaboración propia.

En la tabla aparecen las características articulatorias definidas para estos fonemas consonánticos desde el sistema. Estos fonemas están entre los sometidos a más procesos de variación en el habla, en posición implosiva o de coda silábica en interior y final de palabra, y final absoluto, en la variedad cubana del español.

\section{El fonema /d/}

Gil (2007), refiere que en Cuba es frecuente la pérdida o elisión del fonema consonántico /d/ en posición final de palabra. Entre los procesos de variación de las consonantes obstruyentes oclusivas sonoras más extendidos está «la aparición de las realizaciones aproximantes en posición de ataque silábico. (...) Si ocupan la posición de coda silábica, los procesos de debilitamiento son más importantes y pueden llegar a la pérdida o elisión» (Silverio, 2014: 8).

En relación con la posición intervocálica, es importante señalar que la pérdida o elisión es un fenómeno muy generalizado.

\section{El fonema $/ s /$}

El debilitamiento de /s/ en coda silábica constituye uno de los fenómenos sociolingüísticos más importantes en el conjunto de los procesos del español: la consonante /s/ en coda silábica puede manifestarse como aspirada o asimilada, incluso puede llegar a la elisión. La aspiración de /s/ en contacto con consonantes sordas y sonoras desencadena la asimilación que puede llegar a geminar la consonante; también puede llegar a producirse un único elemento sordo, principalmente en interior de palabra y entre el artículo plural y el nombre que le sigue en la cadena hablada.

La aspiración constituye uno de los fenómenos más representativos del consonantismo en Cuba, sobre todo en la zona dialectal I, igual que la asimilación a la consonante siguiente.

\section{El fonema /I/}

Según la Real Academia Española y la Asociación de Academias de la Lengua Española (2013), existen soluciones africadas [d3] en Cuba. También se documentan manifestaciones de debilitamiento condicionadas por la posición en la sílaba, especialmente en coda silábica: 
- Transformación de [l] en [r]. Su uso se concentra, fundamentalmente, en La Habana, Bahía Honda y Cárdenas. Se puede producir, además, en secuencias tautosilábicas.

- Procesos de aspiración, asimilación hasta la geminación y elisión. Los casos de asimilación y geminación de /// son abundantes en occidente.

- Vocalización (cambio [l] > [i]), nasalización (se convierte en [n]) y palatalización.

\section{Los fonemas $/ r / y / r /$}

Según Silverio (2007), la realización de los fonemas vibrantes de la variedad cubana del español para el occidente (zona dialectal I) se caracteriza por la reducción de la fuerza articulatoria, que provoca una gran variedad de realizaciones, sobre todo en posición implosiva -final o intermedia de palabra. Las variantes que más se registran son: asimilación, favorecida por la posición intermedia; geminación, producto de un proceso de asimilación completo; aspiración, y trueque de [r] por [l], sobre todo al final de palabra.

En esta investigación se asume la aspiración, como pronunciación con flujo de aire más fuerte (Martínez y Fernández, 2007); la asimilación, como pronunciación de un fonema con características del fonema contiguo; la geminación, como la asociación plena a los rasgos del segmento consonántico siguiente, entendida como asimilación completa; la aproximante, como fono resultante de la pronunciación de un fonema sin el contacto completo de los órganos de articulación implicados.

En el análisis de lo suprasegmental en la pronunciación de los profesores de lengua española, niveles A1 y A2, tiene gran importancia el fenómeno de la fragmentación, definida como la realización de pausas no convencionales que rompen la estructura melódica del enunciado, sin una intención comunicativa justificada. Este fenómeno impide o limita la ocurrencia de sinalefa, es decir, la unión de la vocal final - o vocales finales - de una palabra, con la vocal inicial -o vocales iniciales - de la siguiente, de modo que forman una única sílaba a efectos fonéticos y métricos. También entorpece la pronunciación de la palabra fónica, esa que está formada por una palabra (plena o significativa) más los elementos gramaticales átonos que se pronuncian solidariamente con ella.

El grupo fónico está compuesto por una serie de palabras organizadas alrededor de un núcleo marcado por el acento de frase y por una inflexión tonal. Desde el punto de vista de la entonación, el grupo fónico coincide con el contorno entonativo, cuyo núcleo es, igualmente, la inflexión tonal. (Cantero, 2003)

La incidencia del tempo, velocidad relativa con la que se habla cada lengua, en la exposición de la lengua al estudiante, puede determinar el grado de «naturalidad» del modelo lingüístico.

\section{Metodología}

A pesar de que con el habla espontánea se logra una mejor caracterización de la pronunciación de los informantes, en esta investigación las muestras de habla de los profesores fueron tomadas durante las clases, pues la esfera académica es la que interesa a los fines de este trabajo.

En la recogida de la muestra incidieron aspectos negativos, como los relacionados con la calidad de la grabación: interferencias y ruidos ambientales, por lo tanto, fueron desestimados los enunciados poco audibles, de los que tampoco se logró una lectura adecuada en el 
La pronunciación de los profesores de español como lengua extranjera y segunda lengua: la realidad del aula.

espectrograma.

De una población de nueve profesores que regularmente trabajan con los niveles A1 y A2 en la Facultad (cuatro en la Licenciatura en Lengua Española para No Hispanohablantes durante los dos primeros meses del curso - septiembre, octubre-, dos en Preparatoria durante los dos primeros meses del curso -octubre, noviembre-y tres en Curso Corta Duración (CCD) todos los meses), fue empleada una muestra de cinco: dos de la licenciatura en Lengua Española para No Hispanohablantes, dos de Curso Corta Duración (CCD) y un profesor de Curso por Semestre (CS). ${ }^{4}$

La muestra estuvo conformada por cinco informantes de sexo femenino, en un rango de edad entre los 25 y los 60 años. La selección de las informantes estuvo condicionada únicamente por el nivel de lengua que trabajan. Fueron tenidas en cuenta las cinco profesoras que estaban dando clases en el periodo en que se realizaron las grabaciones.

Tabla 2.

Composición de la muestra

\begin{tabular}{|l|c|c|c|c|c|}
\hline & Tipo de curso & $\begin{array}{c}\text { Experiencia } \\
\text { docente en } \\
\text { ELE }\end{array}$ & $\begin{array}{c}\text { Grupo } \\
\text { etario }\end{array}$ & $\begin{array}{c}\text { Zona de } \\
\text { origen }\end{array}$ & $\begin{array}{c}\text { Tiempo en } \\
\text { occidente }\end{array}$ \\
\hline Informante 1 & CCD & 7 & $50-60$ & Occidente & siempre \\
\hline Informante 2 & Preparatoria & 12 & $30-40$ & Occidente & siempre \\
\hline Informante 3 & Licenciatura & 5 & $25-30$ & $\begin{array}{c}\text { Centro- } \\
\text { oriente }\end{array}$ & 5 \\
\hline Informante 4 & $\mathrm{CCD}$ & 5 & $25-30$ & Occidente & siempre \\
\hline Informante 5 & Licenciatura & 11 & $30-40$ & Oriente & 11 \\
\hline
\end{tabular}

Fuente: Elaboración propia.

Por imprevistos, las grabaciones no pudieron ser hechas en el periodo planificado, por lo que las profesoras de la carrera fueron grabadas dando clases en el nivel principiante del Curso Corta Duración (ellas habían trabajado este programa en varias ocasiones); no hubo posibilidad de registrar muestras de lengua oral de las profesoras de Preparatoria, pero el Curso por Semestre abrió un grupo de principiante, en el que trabajó una profesora que tradicionalmente perteneció al colectivo de Preparatoria.

Se recogió, como promedio, una hora de registro sonoro por cada informante. Para esto se solicitó el permiso a los directivos correspondientes de la Facultad y la anuencia de los docentes implicados. Las grabaciones fueron realizadas en los horarios habituales de clases. Se usó una grabadora digital marca Huashengdi, $8 \mathrm{G}$ de referencia NWZ-B142F, programada con una frecuencia de muestreo de $44100 \mathrm{~Hz}$ en sonido monofónico. Los registros fueron almacenados en formato WAV.

Las grabaciones fueron hechas los días 17 (aula 8), 20 (aula 12) y 26 de abril de 2018 (aula 11); 3 (aula 8) y 8 de mayo del mismo año (aula 8), en la Facultad de Español para No Hispanohablantes de la Universidad de La Habana. Las actividades académicas que fueron

\footnotetext{
${ }^{4}$ Este curso está dirigido a estudiantes de universidades estadounidenses con las que la Universidad de La Habana tiene convenio. Durante un semestre reciben, en la facultad, Lengua española, Práctica integral de la lengua española y otras asignaturas relacionadas con la lengua y la cultura cubanas, según los intereses de los estudiantes. Regularmente vienen con nivel intermedio y superior.
} 
visitadas correspondieron al nivel principiante del Curso de Corta Duración, primera semana, y una del Curso por Semestre, cuarta clase. Esto permitió obtener muestras de lengua hablada durante noventa minutos, que es el tiempo de duración de dos horas clase.

Se realizaron las transcripciones de las intervenciones de las profesoras, en documentos con formato TextGrid, y luego se separaron las palabras que incluían los fonemas /s/, /d/, II/, /r/ y /r/, en los contextos objeto de análisis: posición final de sílaba (intermedia y final de palabra, absoluta o no). En el caso de /d/, además, posición intervocálica. Para la descripción, se llevó a un documento Excel mediante la transcripción fonética semiestrecha (tiene en cuenta los alófonos), a partir del Alfabeto Fonético Internacional (AFI).

Para esta investigación se concibió la descripción articulatoria a partir de la percepción auditiva, que sigue siendo la base de la fonética elemental tradicional. Se escuchó en repetidas ocasiones cada uno de los fonemas insertados en su contexto. Así se logró identificar el modo y la zona de articulación, y la clasificación según la acción de las cuerdas vocales y la acción del velo del paladar.

El programa Praat, en su versión 6.0.36, se empleó para la transcripción de toda la grabación y el análisis de aquellos segmentos de la cadena hablada en los que la percepción sugería que había variaciones. Se trabajó con una frecuencia de señal de muestreo de 5,000 $\mathrm{KHz}, 16$ bits, mono.

Finalmente, se hizo un conteo de la cantidad de segmentos que resultaron de todos los informantes y se clasificaron en nuevas tablas que permitieron ver la cantidad de segmentos de un contexto específico producido por cada informante, así como las diferentes realizaciones 0 variaciones fonéticas.

No se tuvo en cuenta la distinción entre los fonemas $/ r / \mathrm{y} / \mathrm{r} /$ debido a que, en la posición que se analiza, se neutralizan; es decir, la realización de uno u otro no afecta el mensaje ni desde el punto de vista semántico ni desde la expresión.

\section{Aspectos generales de la pronunciación de las informantes}

Las características de la pronunciación de cada informante que se exponen a continuación son el resultado de la percepción auditiva del investigador. De forma general, las informantes hacen un poco más lento el tempo de la lengua española, sin que ello afecte la entonación, por lo que no se considera una alteración al modelo de lengua didácticamente relevante. Este comentario no se hace sobre la base de mediciones, sino a partir de la observación y la comparación con el tempo de las informantes en diferentes momentos de la clase y fuera de ella.

Informante 1: Se encuentra en el grupo etario 50-60 y es oriunda de la región occidental del país. Se caracteriza por hablar muy despacio y pronunciar cada palabra, con lo que se afecta el ritmo y el acento del enunciado. En ocasiones divide en sílabas para garantizar que el estudiante identifique todos los sonidos. Esta estrategia puede ser válida siempre y cuando la profesora haya ofrecido el modelo real. En la grabación no se identifican casos de sinalefa. La informante solo recupera el tempo real de la lengua cuando se dirige al estudiante sin que le interese que la comprenda (información que se aleja de su objeto de estudio), o cuando supone que el estudiante ya comprendió el asunto. Contradictoriamente, fue la que más variantes alofónicas realizó.

Informante 2: Se encuentra en el grupo etario $30-40$ y es oriunda de la región occidental 
del país. Su pronunciación es expresión del ambiente distendido, de confianza, que impera en el aula; natural. La profesora ríe oportunamente. La informante aprovecha las posibilidades de sinalefa y los enlaces consonante-vocal, propios de las palabras fónicas. Conserva el acento de grupo y el tempo de la lengua.

Informante 3: Se encuentra en el grupo etario $25-30$ y es oriunda de la región centrooriental del país. Tiene una pronunciación que se caracteriza por gran estabilidad en la realización de los sonidos, propio de Camagüey. La informante aprovecha las posibilidades de sinalefa y los enlaces consonante-vocal, propios de las palabras fónicas. Conserva el acento de grupo y el tempo de la lengua.

Informante 4: Se encuentra en el grupo etario 25-30 y es oriunda de la región occidental del país. Tiene una pronunciación que se caracteriza por gran estabilidad en la realización de los sonidos.

Con frecuencia, sobre todo cuando da las órdenes o pregunta, fragmenta el enunciado:[¿kómo/está/estér?//],[enespañól/kwándopreyuntámosporlosáños/][bánaleér/kompl éto/eldjáloyo//].

Predomina la intención de pronunciar cada sonido, lo que lleva a la informante a no aprovechar las posibilidades de sinalefa ([kómo/está/estér//]) y los enlaces consonante-vocal, propios de las palabras fónicas ([enespañól], [porlosáños]), aunque la informante conserva el acento de grupo y el tempo de la lengua.

Informante 5: Se encuentra en el grupo etario $30-40$ y es oriunda de la región oriental del país. Es significativo el énfasis que la informante hace en la pronunciación de los fonemas $|\mathrm{s} /|$,$r / y |r|$ en coda silábica, sobre todo de las vibrantes. Es propia del habla oriental la pronunciación plena de las consonantes vibrantes, no así la de la/s/. En este caso, la informante, como representante de la norma culta de la variedad cubana del español, profesora de lengua en el occidente del país desde hace aproximadamente ocho años, pone cuidado en la realización del fonema. Aunque pronuncia cada palabra del enunciado, lo fragmenta, enseguida lo retoma con el ritmo adecuado ([kwántos/áños/tjéne//], [kwántosáñostjéne//]). Con frecuencia alarga una palabra del enunciado mediante el alargamiento de la vocal (que lleva el acento de intensidad, si es una palabra plena) con la intención de llamar la atención del estudiante sobre determinado aspecto ([entónses/bámosa trabajárkó:n/éstetéksto//]). La informante aprovecha las posibilidades de sinalefa ([ésyórdo:/oésfláko//]) y los enlaces consonante-vocal, propios de las palabras fónicas. Conserva el acento de grupo y el tempo de la lengua. Su entonación está marcada por la variedad oriental de la lengua.

\section{Fonemas $/ s /, / d /$, III, $/ r /$ y $/ r /$}

De las grabaciones se obtuvo un corpus conformado de la forma siguiente: 
Tabla 3.

Composición del corpus

\begin{tabular}{|c|c|c|c|c|c|c|c|}
\hline Fonema & Contexto & $\begin{array}{c}\text { Informante } \\
1 \\
\end{array}$ & $\begin{array}{c}\text { Informante } \\
2 \\
\end{array}$ & $\begin{array}{c}\text { Informante } \\
3 \\
\end{array}$ & $\begin{array}{c}\text { Informante } \\
4\end{array}$ & $\begin{array}{c}\text { Informante } \\
5\end{array}$ & Totales \\
\hline \multirow{4}{*}{$/ \mathrm{d} /$} & $\begin{array}{l}\text { coda } \\
\text { silábica } \\
\text { interior de } \\
\text { palabra }\end{array}$ & 14 & 0 & 0 & 0 & 2 & 16 \\
\hline & $\begin{array}{l}\text { final de } \\
\text { palabra }\end{array}$ & 32 & 7 & 4 & 7 & 8 & 58 \\
\hline & $\begin{array}{l}\text { final } \\
\text { absoluto }\end{array}$ & 23 & 1 & 0 & 9 & 0 & 33 \\
\hline & $\begin{array}{l}\text { posición } \\
\text { intervocálic } \\
\text { a }\end{array}$ & 108 & 73 & 55 & 85 & 48 & 369 \\
\hline \multicolumn{2}{|c|}{ Totales } & 177 & 81 & 59 & 101 & 58 & 476 \\
\hline \multirow{3}{*}{$|s|$} & $\begin{array}{l}\text { coda } \\
\text { silábica } \\
\text { interior de } \\
\text { palabra }\end{array}$ & 151 & 150 & 90 & 91 & 88 & 570 \\
\hline & $\begin{array}{l}\text { final de } \\
\text { palabra }\end{array}$ & 552 & 256 & 319 & 443 & 415 & 1985 \\
\hline & $\begin{array}{l}\text { final } \\
\text { absoluto }\end{array}$ & 75 & 37 & 16 & 43 & 39 & 210 \\
\hline \multicolumn{2}{|c|}{ Totales } & 778 & 443 & 425 & 577 & 542 & 2765 \\
\hline \multirow{3}{*}{ /I/ } & $\begin{array}{l}\text { coda } \\
\text { silábica } \\
\text { interior de } \\
\text { palabra }\end{array}$ & 9 & 27 & 7 & 14 & 17 & 74 \\
\hline & $\begin{array}{l}\text { final de } \\
\text { palabra }\end{array}$ & 163 & 78 & 50 & 127 & 118 & 536 \\
\hline & $\begin{array}{l}\text { final } \\
\text { absoluto }\end{array}$ & 2 & 0 & 0 & 0 & 0 & 2 \\
\hline \multicolumn{2}{|c|}{ Totales } & 174 & 105 & 57 & 141 & 135 & 612 \\
\hline \multirow{3}{*}{$|r / \mathrm{yr}|$} & $\begin{array}{l}\text { coda } \\
\text { silábica } \\
\text { interior de } \\
\text { palabra }\end{array}$ & 214 & 58 & 82 & 83 & 124 & 561 \\
\hline & $\begin{array}{l}\text { final de } \\
\text { palabra }\end{array}$ & 115 & 45 & 57 & 61 & 82 & 360 \\
\hline & $\begin{array}{l}\text { final } \\
\text { absoluto }\end{array}$ & 14 & 3 & 1 & 4 & 0 & 22 \\
\hline \multicolumn{2}{|c|}{ Totales } & 343 & 106 & 140 & 148 & 206 & 943 \\
\hline
\end{tabular}

Fuente: Wang (2018: 34). 
La pronunciación de los profesores de español como lengua extranjera y segunda lengua: la realidad del aula.

Aunque son explicados algunos casos de variaciones, se parte de que en español existe una tendencia a la sílaba abierta, por lo que la consonante implosiva o en coda silábica está muy propensa a los procesos de debilitamiento articulatorio, a procesos de variación.

En todos los casos analizados se observó que los informantes realizan los fonemas en la zona de articulación definida para cada uno y mantienen el rasgo oral.

\section{El fonema $/ \mathbf{s} /$}

En relación con el fonema /s/ en posición implosiva o coda silábica, se distinguen dos casos lingüísticamente significativos: la aspiración y la omisión, sobre todo el segundo. Esto no se corresponde con la tendencia general de la pronunciación del español en la zona geolectal del Caribe, por tanto, de Cuba: la aspiración como fenómeno más recurrente.

La variante de mayor frecuencia en las cinco informantes fue la fricativo alveolar sordasibilante [s]. El fonema /s/ incluye varias clases de sibilantes (convexas, cóncavas, largas, cortas, parcialmente sonorizadas, entre otras).

El comportamiento alofónico se manifiesta de esta manera en las cinco informantes.

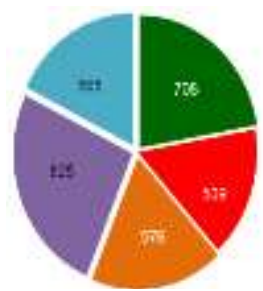

Gráfico 1

Realizaciones plenas de $/ s /$

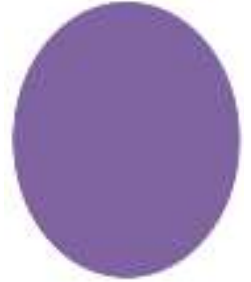

Gráfico 2

Realizaciones

asimiladas de

$|\mathbf{s}|$

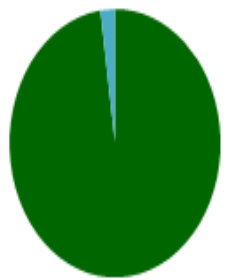

Gráfico 3

Realizaciones

aspiradas de $\mid \mathbf{s} /$

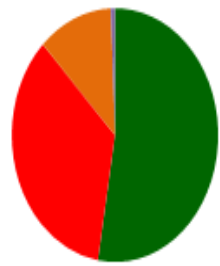

Gráfico 4

Omisiones de /s/

Informante 1

Informante 2

Informante 3

Informante 4

Informante 5

Fuente: Wang (2018).

Como demuestran los gráficos, el mayor porcentaje lo alcanzó el fonema /s/ realizado plenamente, y la mayor incidencia de variaciones se encontró en las informantes 1 y 2, ambas de la región occidental y de mayor edad.

Los menores índices de omisiones pertenecen a las informantes 3, 4 y 5 , dos de la región centro-oriental y oriental, y una de la occidental, quienes son exponentes de una gran estabilidad en la realización de los fonemas.

Los datos demuestran que la elisión del fonema /s/ en el habla de las profesoras es más avanzada que su aspiración. Los dos procesos son variables y los dos se ven condicionados, entre otros factores, por el contexto fonológico. La norma, por lo general, es la realización plena del fonema /s/ y su alófono aspirado. 


\section{El fonema $/ \mathrm{d} /$}

En una evaluación del corpus de habla se percibe la variante oclusiva del fonema, en posición intervocálica, contexto no habitual para esta variante. El fonema oclusivo dental sonoro /d/ tiene como realizaciones varios alófonos: el oclusivo, dental, sonoro, oral, [d], que aparece en inicial absoluta y después de n, s, I (ondulado); el aproximante, dental, sonoro, oral [ð], en posición intervocálica; el oclusivo, dental, sordo, oral (como/t/, en posición final). También puede ser aspirado [h] u omitido en posición final absoluta.

Dieciséis realizaciones de /d/ $(3,36 \%)$ en coda silábica, interior de palabra, fueron manifestaciones oclusivas, dentales, sordas, orales (como [t]). Se evidencia un fenómeno de neutralización a favor del fonema sordo.

De forma general, las realizaciones descritas por la literatura especializada ([d] y [ð]]) obtuvieron los más altos porcentajes. Las variaciones se encontraron en los informantes 1, 3 y 4 , aunque los números no son lingüísticamente significativos.

El proceso de debilitamiento $[\mathrm{d}] \rightarrow[ð] \rightarrow[\varnothing]$ es de origen interno y está presente en la mayoría de las comunidades dialectales. Llama la atención que el $11,65 \%$ del total de realizaciones intervocálicas corresponde al alófono oclusivo [d].

Este comportamiento está relacionado con la pronunciación fragmentada, de manera intencional, para que el estudiante perciba todos los sonidos de la palabra. El mayor porcentaje corresponde a la informante 1, lo que es consecuente con lo expuesto sobre su pronunciación. También la informante 5 fragmenta, con el mismo objetivo, aunque repite el enunciado correctamente. En su caso se identificaron ocho realizaciones oclusivas.

Se identificó la pérdida o elisión del fonema consonántico /d/ en posición final de palabra, aunque no con un porcentaje significativo, en las informantes 1,3 y 4.

\section{El fonema /II}

Las variaciones no fueron significativas y los porcentajes no son lingüísticamente relevantes. El comportamiento alofónico se manifiesta de la manera siguiente en las cinco informantes.

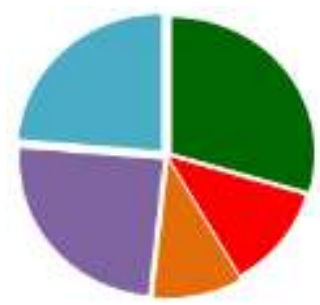

\section{Gráfico 5. Realizaciones plenas de /I/}

Fuente: Wang (2018). 
La pronunciación de los profesores de español como lengua extranjera y segunda lengua: la realidad del aula.

El fonema /// fue uno de los que mejor comportamiento registraron. Los mayores porcentajes corresponden a realizaciones laterales plenas. Los casos de relajamiento, aunque los porcentajes no son significativos, se produjeron en posición intermedia de palabra.

\section{Los fonemas $/ r /$ y $/ \boldsymbol{r} \mid$}

El fonema /r/ se realizó en todos los casos como vibrante, ya sea simple o múltiple. Aunque se identificaron varios casos de realizaciones aproximantes, no se tuvo en cuenta la distinción. Como es sabido, las vibrantes están entre los sonidos más inestables, sujetos a múltiples procesos de variación, pero a los objetivos de esta investigación solo resultan pertinentes aquellos que afectan la pronunciación como modelo en la clase de español como lengua extranjera (ELE). Las cinco informantes mostraron el mismo comportamiento.

Las vibrantes se caracterizaron por presentar periodos oclusivos (interrupciones de energía) muy breves, que corresponden a las pequeñas oclusiones producidas en el tracto vocal. Cada una de esas oclusiones dio origen a su propia explosión, seguida de un periodo vocal, todo ello muy breve.

\section{Regularidades}

El análisis del comportamiento de los fonemas objeto de estudio permitió identificar las regularidades siguientes:

1. El contexto fonológico incide en la realización plena o no de los fonemas /s/, /d/, II/, Ir/ y $\mid \mathrm{r} /$.

2. La informante 1 fue la que más variantes alofónicas utilizó, especialmente las aspiraciones y en algunos casos, omisiones.

3. Aunque en menor medida que la informante 1 , la 2 presentó variaciones en los fonemas $/ \mathrm{s} / \mathrm{y} / \mathrm{d} /$, no asi en $/ / / \mathrm{y}$ en las vibrantes.

4. De forma general, las cinco informantes evidenciaron altos porcentajes de realizaciones plenas de los fonemas estudiados.

5. Las variantes alofónicas encontradas más recurrentes resultaron del fonema $/ \mathrm{s} /$ : omisiones y aspiraciones.

6. Los casos de aspiraciones del fonema /s/ se encontraron en posición de coda silábica, sobre todo en los pronombres demostrativos: esta, este, estos, este.

7. Los casos de elisión de /s/ son más frecuentes en posición final de palabra que en posición de coda silábica, interior de palabra.

8. El fonema /s/ en posición intervocálica en juntura de palabras se manifiesta a través de los fonos [s] y [h]. En los casos encontrados se pudo apreciar una leve aspiración.

9. Entre las diferentes clases de palabras terminadas en /s/ lexical, se evidencia una variación mayor, aparentemente significante en las palabras polisilábicas. Estas son más propensas a la elisión que las monosilábicas (es, tres, dos, etcétera), con excepción de los artículos.

10. La frecuencia léxica contribuye a las variaciones de la $/ \mathrm{s} /$. Mientras más alta la frecuencia de aparición de una palabra en el discurso, mayor probabilidad de aparición de procesos de debilitamiento.

11. Las omisiones de /d/ ocurren, generalmente, en posición final absoluta de palabra.

12. Predomina la realización aproximante de la consonante /d/ intervocálica. 
13. La realización oclusiva de la consonante /d/ intervocálica tiene una función enfática.

14. Los fonemas vibrantes y el lateral /// tienen gran estabilidad, acorde con lo descrito para el sistema.

\section{Conclusiones}

El análisis de las muestras de lengua oral de cinco profesoras de ELE y L2 de los niveles A1 y A2 de la FENHI permitió comprobar que las docentes constituyen un modelo lingüístico adecuado para los estudiantes. Ellas tienen una pronunciación caracterizada por la estabilidad en la realización de los sonidos, aunque tienden a fragmentar el enunciado a favor de la comprensión de los estudiantes, lo que limita la pronunciación correcta de las palabras fónicas, pues desaprovechan posibilidades de sinalefa y enlaces consonante-vocal, propios de las palabras fónicas. La disminución del tempo - velocidad relativa con que se habla- puede ser una solución más acertada en estos casos.

El análisis de las producciones de los fonemas consonánticos $/ \mathrm{s} /, / \mathrm{d} /, / \mathrm{ll}, \mathrm{rr} / \mathrm{y} / \mathrm{r} / \mathrm{del}$ español, reveló que las informantes:

1. Mantienen la zona de articulación y el carácter oral descritos para los fonemas consonánticos estudiados.

2. Los principales procesos de variación están relacionados con los fonemas $/ \mathrm{s} / \mathrm{y} / \mathrm{d} /$ : aspiración y elisión en el primer caso; realización aproximante (posición intervocálica) y como oclusiva sorda en el segundo (coda silábica).

3. Los casos de variantes alofónicas que se encontraron tuvieron mayor incidencia en los pronombres demostrativos y en los adjetivos con terminación -ado.

4. La frecuencia léxica tuvo que ver con las variaciones. Mientras más alta fue la frecuencia de aparición de una palabra en el discurso, mayor probabilidad hubo de que en dicha palabra ocurriera un debilitamiento.

Esto corrobora lo descrito en la bibliografía especializada en relación con los procesos de variación de los fonemas consonánticos estudiados.

\section{Referencias bibliográficas}

Cantero, Francisco José (2003). Fonética y didáctica de la pronunciación. En A. Mendoza (coordinador). Didáctica de la lengua y la literatura para primaria. Prentice Hall. España.

Choy López, Luis Roberto (1988). Consonantismo en el habla culta de Ciudad de La Habana y Santiago de Cuba. Revista Islas 91, sep.-dic. Cuba (pp. 130-143).

Costa, Manuel (1993). Algunos fenómenos articulatorios de la cadena hablada. Revista Islas 106, sep.-dic. Cuba (pp. 101-104).

Costa, Manuel y Susana Carreras (1980). Algunas características acústico-articulatorias de la vibrante múltiple en el español de Cuba. Revista Islas 65, abril. Cuba (pp. 99-114).

D'Introno, Francesco, Enrique del Teso y Rosemary Weston (1995). Fonética y fonología actual del español. Revista Cátedra, 478. España (pp. 450-459).

Dohotaru, Puica (1998/1999). Condicionamiento lingüístico y social de la variación de -/R/ en el habla de habaneros universitarios. Anuario L/L Estudios Lingüísticos 29-30. Cuba (pp. 51-76). 
La pronunciación de los profesores de español como lengua extranjera y segunda lengua: la realidad del aula.

García González, José (1980). Acerca de la pronunciación de R y L implosivas en el Español de Cuba: variantes e influencias. Revista Islas 65, en.-abr. Cuba (pp. 115-127).

Gil, Juana (2007). Fonética para profesores de español: de la teoría a la práctica. Editorial Arco/Libros S.A. España.

Hernández, Shadia (2011). La corrección fonética y el karaoke como herramienta motivadora. Propuesta de un sistema de ejercicios. Tesis de especialidad, Facultad de Español para No Hispanohablantes, Universidad de La Habana. Cuba.

Isbasescu, Cristina (1968). El español de Cuba. Observaciones fonéticas y fonológicas. Sociedad Rumana de Lingüística Románica. Rumania.

Lahera, Yunier (2011). El silabismo y su influencia sobre los segmentos: estudio de las aproximantes en estudiantes sinohablantes. Tesis de maestría. Facultad de Español para No Hispanohablantes, Universidad de La Habana. Cuba.

Marco Común Europeo de Referencia para las Lenguas (MCER) (2002). Traducción y adaptación española del European Framework, Consejo de Europa y Dirección Académica del Instituto Cervantes. En www.cvc.cervantes.es/obref/marco. Consulta: 05/01/2020

Martínez, Eugenio y Ana María Fernández (2007). Manual de fonética española. Ariel, España. Mingjin, Qiu (2016). Descripción articulatoria de las consonantes $/ \mathbf{b} /, / \mathbf{d} / \mathbf{y} / \mathbf{g} / \mathbf{d e l}$ español realizadas por estudiantes chinos. Tesis de maestría. Facultad de Español para No Hispanohablantes, Universidad de La Habana. Cuba.

Montero Bernal, Lourdes (2007). Zonificación geolectal de Cuba desde el punto de vista fonético.

En S. Valdés et al. Visión geolectal de Cuba, Peter Lang. Internationaler Verlag der Wissenschften. Band 8, Suiza (pp. 33-51.)

Puig, Yareira (2004). Nuevas posibilidades para la intensificación del proceso de enseñanza del español como lengua extranjera en los Cursos de Corta Duración: su aplicación en una serie de libros de texto. Tesis de doctorado, Facultad de Lenguas Extranjeras, Universidad de La Habana. Cuba.

Quilis, Antonio (1993). Tratado de fonología y fonética españolas. Editorial Gredos, España. Real Academia Española y Asociación de Academias de la Lengua Española (2013). Nueva gramática de la lengua española. Fonética y fonología. Planeta, Argentina.

Silverio, Tania (2007). La realización de las vibrantes españolas por estudiantes chinos.

Tesis de maestría, Facultad de Artes y Letras, Universidad de La Habana. Cuba.

Silverio, Tania (2014). Estrategia linguodidáctica para perfeccionar la enseñanza de la pronunciación en las clases de español como lengua extranjera. Tesis de doctorado, Facultad de Lenguas extranjeras, Universidad de La Habana. Cuba.

Tristá, Antonia María y Sergio Valdés (1978). El consonantismo en el habla popular de La

Habana, Editorial de Ciencias Sociales, Cuba.

Valdés, Sergio (2007). El poblamiento de Cuba y las áreas geolectales. En S. Valdés et al.:

Visión geolectal de Cuba, Peter Lang. Internationaler Verlag der Wissenschften. Band 8, Suiza (pp. 8-32).

Valdés, Sergio (2015). La hispanización de América y la americanización de la lengua española. Editorial UH, Cuba.

Wang, Ye (2018). La pronunciación de los profesores de español como lengua extranjera en los niveles A1 y A2: el modelo. Tesis de maestría, Facultad de Español para No Hispanohablantes, Universidad de La Habana. Cuba. 
Wei, Jungiao (2016). El perfeccionamiento de la pronunciación del español para favorecer el desarrollo de la expresión oral de sinohablantes en la asignatura Lengua y Comunicación I. Tesis de maestría, Facultad de Español para No Hispanohablantes, Universidad de La Habana. Cuba.

Wei, Jungiao, Dell Liu, y Sun, Zin (2013). Conjunto de ejercicios para el tratamiento de la corrección fonética en estudiantes chinos tercer año de Pedagogía en Humanidades. Tesis de licenciatura, Universidad de Ciencias Pedagógicas Enrique José Varona, Cuba. 